\title{
Reproducibility and accuracy of measurements with a hand held autorefractor in children
}

\author{
Erin M Harvey, Joseph M Miller, L Keith Wagner, Velma Dobson
}

Department of

Ophthalmology,

University of Arizona,

Tucson, USA

E M Harvey

J M Miller

L K Wagner

V Dobson

Optical Sciences

Center, University of

Arizona, Tucson, USA

J M Miller

Department of

Psychology, University

of Arizona, Tucson,

USA

V Dobson

Correspondence to: Erin Harvey, University of Arizona College of Medicine, Department of

Ophthalmology, $1801 \mathrm{~N}$ Campbell Avenue, Tucson, AZ 85719 , USA.

Accepted for publication 10 June 1997

\begin{abstract}
Aim-To determine reproducibility and accuracy of the Nikon Retinomax autorefractor when used with children who were made cycloplegic.

Methods-Autorefraction and retinoscopy or subjectively refined retinoscopy (where, under the patient's direction, the refraction was varied until the best visual acuity was achieved) were performed on the right eye of 47 children, age 11-93 months. Autorefraction was performed using the Nikon Retinomax, which provides up to eight measured values of refractive error and one representative measurement of refractive error.

Results-Autorefractor measurements were successfully obtained from $7 / 9$ children age 3 years or younger, and from all older children. Vector methods were used to calculate differences. Retinomax reproducibility averaged $0.43 \mathrm{D}$. Unbiased Retinomax and retinoscopy measurements differed by an average of $0.82 \mathrm{D}$. Unbiased Retinomax and subjectively refined retinoscopy differed by an average of $1.03 \mathrm{D}$.

Conclusions-Reproducibility of Retinomax measured values in children is comparable with reproducibility of retinoscopy, subjective refraction, and autorefraction measurements in adults. Agreement between Retinomax and retinoscopy and agreement between Retinomax and subjective refinement in children is comparable with agreement between autorefraction and subjective refraction in adults. The study indicates that the Retinomax is a useful instrument for measuring refractive errors in young children.

(Br F Ophthalmol 1997;81:941-948)
\end{abstract}

High refractive error (for example, anisometropia, astigmatism, hyperopia) in early childhood can lead to amblyopia. ${ }^{1-4}$ There is evidence that amblyopia can be prevented if children at risk receive optical correction at an early age..$^{5-8}$ Thus, it may be valuable to identify and correct children for high refractive error as early as possible.

The traditional method for detecting refractive errors in preschool age children involves cycloplegic or non-cycloplegic retinoscopy. Although skilled retinoscopists can provide reliable and valid measures of refractive error in children, ${ }^{39}$ retinoscopy is subject to interobserver variation. ${ }^{10}{ }^{11}$ For this reason, retino- scopy measurements are not an appropriate 'gold standard' for evaluating measurements of refractive error. Recently, there has been an emphasis on the development of measurement tools that are free of operator bias, can be used by lay individuals, and provide a 'gold standard' for retinoscopy measurements. One such tool is the Nikon Retinomax autorefractor (Nikon, Melville, NY, USA), a hand held instrument that measures refractive error. To date, there are no reports of the efficacy, validity, or reliability of the Retinomax for the measurement of refractive error in children.

The aim of this study was to determine if the Retinomax can be used successfully with children under cycloplegic conditions, to examine the reproducibility of the Retinomax in measuring refractive error in children, and to assess the agreement between the Retinomax and retinoscopy and the agreement between the Retinomax and subjective refinement. For each eye, the Retinomax provides up to eight measured values, and one representative measurement of refractive error. We evaluated reproducibility of Retinomax measurements through analysis of Retinomax measured values of refractive error. We evaluated measurement agreement by comparing Retinomax representative measurements with retinoscopy and subjective refinement measurements.

\section{Methods}

MATERIALS

Retinomax autorefractor

The Nikon Retinomax is a hand held instrument for measuring refractive error. The instrument is designed for easy use by individuals with minimal ophthalmic experience. The Retinomax provides up to eight measured values of refractive error (including sphere, cylinder, and axis) and then determines a single best representative measure based on the measured values. The algorithm used by the manufacturer for determination of the representative measurement is not published.

\section{SUBJECTS}

Subjects were 47 consecutive patients, under the age of 8 years, seen in the paediatric ophthalmology clinic at the University of Arizona. None of the subjects had amblyopia or other cause of decreased visual acuity that precluded fixation with the right eye, and none exhibited any ocular pathology other than refractive error. This study was approved by the University of Arizona Institutional Review 
Board (IRB). Written informed consent was obtained from parents of all children, and verbal assent was obtained from older children.

\section{PROCEDURE}

In order to achieve cycloplegia, each child was first given proxymetacaine (proparacaine) $0.5 \%$, followed 30 seconds later by a single drop of cyclopentolate $1 \%$ instilled in the inferior cul de sac. The child was held with the eyelid open and the pooled drop in contact with the cornea for approximately 1 second, and then the upper eyelid was lifted, allowing the pooled drop to collect under the upper eyelid. Twenty to 30 minutes later, dynamic retinoscopy was performed to determine adequacy of cycloplegia. Dynamic retinoscopy was performed by observing the retinoscopic reflex while the child was viewing a distant television monitor displaying a cartoon, stopping the cartoon, and then directing the child to attend to a finger puppet held alongside the retinoscope. Evidence of inadequate cycloplegia was a shift in the retinoscopic reflex as viewing distance changed. In no instance was it necessary to instil additional drops.

A certified ophthalmic technician (LKW) then attempted to obtain a Retinomax measurement from the right and left eyes of each subject. Older children were asked to remain still and to fixate on the Retinomax internal fixation target as the examiner held the Retinomax in perpendicular alignment. For younger children, a parent held the child while the examiner aligned the Retinomax. Once fixation was achieved, the examiner attempted a measurement. For children who initially demonstrated little interest in the fixation target, a modified technique was used. Firstly, a small finger puppet was used to attract the child's attention. Then, all room lights were turned off and the Retinomax was immediately positioned in front of the child. Thus, the only visual stimulus present, the fixation target of the Retinomax, attracted the child's attention, and the measurement was made. The Retinomax provided a printout for each eye that included several measured values of refractive error and a single representative measurement of refractive error. For the right eye, we obtained two measured values for one subject, three measured values for one subject, six measured values for four subjects, and eight measured values for all other subjects.

Immediately after autorefraction, an ophthalmologist (JMM) performed retinoscopy followed by subjective refinement, when possible. Subjective refinement was achieved by varying the refraction under the patient's direction until best visual acuity was achieved. The ophthalmologist was masked to the results of the autorefraction at the time retinoscopy with or without subjective refinement was performed. For subjects with whom subjective refinement was performed, the ophthalmologist recorded a single best estimate of refractive error for each eye. For subjects who were assessed with retinoscopy, the ophthalmologist recorded the phoropter reading at neutralisation.
DATA ANALYSES

In order to avoid violations of independence of measurements that arise when data from both eyes of subjects are included in analyses, only data from the right eye of each subject were included. The data relevant to the following analyses were each subject's Retinomax measured values, Retinomax representative measurement, and retinoscopy or subjectively refined measurement of refractive error.

Analysis of the reproducibility and accuracy of refractive error data is complicated by the interaction of sphere, cylinder, and axis measurements. Small axis variations of large cylinder powers can produce the same dioptric blur as large axis variations of small cylinder power. That is, variation of either sphere or cylinder power can produce variation in the net dioptric power. Analysis of the variation of astigmatic power is best accomplished with the vector method described by Long ${ }^{12}$ and modified by Harris. ${ }^{13}$ Using this method, each observation of sphere, cylinder, and axis is converted into a single point in dioptric three dimensional space. The advantage of this method is that the deviation for each measurement can be derived in units of dioptres that are related simultaneously to the sphere, cylinder, and axis of the measurements. Repeated observations result in a cluster of points occurring in this three dimensional space. For each subject, the Retinomax measured values of refractive error (usually eight measured values per subject), the Retinomax representative measurement, and the retinoscopy or subjective refinement measurement of refractive error were converted to a value representing a point in dioptric three dimensional space using the method of Harris. ${ }^{13}$

\section{REPRODUCIBILITY}

Reproducibility of Retinomax measurements was evaluated through the analysis of the Retinomax measured values provided for each subject. The mean of the measured values was calculated according to the method of Harris ${ }^{13}$ for each subject, and the vector dioptric distance between each estimate and the mean of that subject's measured values was calculated. This yielded a dioptric error value for each estimate of refractive error. The mean of the dioptric errors was calculated for each subject, and the grand mean dioptric error was calculated for all subjects, for subjects who underwent retinoscopy, and for subjects who permitted subjective refinement. For each group and for subjects overall, Spearman correlation was used to evaluate the relation between reproducibility of measurements (dioptric error) and subject age, amount of spherical power, and amount of astigmatism. Spherical power and astigmatism values for correlations were obtained from subjects' retinoscopy or subjective refinement measurements.

\section{MEASUREMENT BIAS}

Since individual retinoscopists, refractionists, and the Retinomax may have a consistent measurement bias, we determined the bias 
between retinoscopy and Retinomax representative measurements, and the bias between subjective refinement and Retinomax representative measurements. For subjects who underwent retinoscopy, each subject's Retinomax representative measurement (sphere, cylinder, axis) was subtracted from his/her retinoscopy measurement-that is, the phoropter reading at neutralisation, using vector methods. For subjects who permitted subjective refinement, each subject's Retinomax representative measurement was subtracted from his or her subjectively refined retinoscopy measurement using vector methods. For each group (retinoscopy/subjective refinement), the overall mean of these values was calculated to determine the amount and direction of measurement bias.

While we assume the best subjective refinement under cycloplegia represents an unbiased estimate of the subject's refractive error, we cannot make that assumption for retinoscopy. In retinoscopy, there is large variation between retinoscopists in both spherical measurement accuracy (arising from working distance variation from the typical two thirds of a metre) and cylinder (arising from off axis measurement). Therefore, the measurement bias between retinoscopy and Retinomax measurements represents an 'ideal retinoscopy lens' that, when held before the subject, would best correct for retinoscopy working distance and off axis alignment, as well as any systematic errors that might be present within the Retinomax.

\section{MEASUREMENT AGREEMENT}

We evaluated the agreement between Retinomax representative measurements and retinoscopy measurements at neutralisation, and agreement between Retinomax representative measurements and subjective refinement. In order to correct for systematic measurement bias, the mean bias for retinoscopy was subtracted from retinoscopy measurements, and the mean bias for subjective refinement was subtracted from subjective refinement measurements (see Measurement bias, above). This correction yielded a means of comparing the Retinomax with retinoscopy and subjective refinement free of systematic bias. The corrected retinoscopy and subjective refinement measurements were used in analyses of measurement agreement.

For each group, the absolute magnitude of vector dioptric difference between each subject's Retinomax representative measurement and that subject's retinoscopy with or without subjective refinement derived estimate of refractive error was calculated, and the mean of the absolute differences was calculated for retinoscopy and subjective refinement groups. Spearman correlation was used to determine the relation between measure of agreement and subject age, amount of spherical power, and amount of astigmatism for each group. Spherical power and astigmatism values for correlations were obtained from subjects' retinoscopy or subjective refinement measurements.
Use of absolute values in the calculation of deviation resulted in positive mean dioptric deviations. If absolute values had not been used, the mean dioptric difference between the Retinomax and Retinoscopy measurements and between the Retinomax and subjective refinement measurements would have been zero, since we subtracted off the bias between methods of measurement before calculating mean dioptric deviation. Thus, the mean dioptric deviation represents the average difference in any vector direction between measurements obtained by two methods (Retinomax and retinoscopy or Retinomax and subjective refinement).

\section{Results}

SUCCESS IN OBTAINING MEASUREMENTS

The ophthalmic technician was successful in obtaining Retinomax measurements from seven of nine subjects who were age 3 years or younger, and from all 38 older subjects. The two subjects from whom we could not obtain measurements were 11 and 14 months old. Obtaining measurements from children less than 3 years of age was more difficult, as they tended to try to turn away from the instrument.

The ophthalmologist was successful in completing retinoscopy in 25 subjects, and subjectively refined retinoscopy in 22 subjects. Overall, we obtained Retinomax and retinoscopy measurements in 23 children (retinoscopy group, mean age 4.1 (SD 1.5) years), Retinomax and subjective refinement measurements in 22 children (subjective refinement group, mean age 6.6 (SD 1.0) years), and only retinoscopy measurements in two children (mean age 1 year). Subjects in the retinoscopy group were significantly younger than subjects in the subjective refinement group $(\mathrm{t}(43)=$ $6.54, \mathrm{p}<0.0001)$.

SUMMARY OF RESULTS FROM INDIVIDUAL SUBJECTS

In Tables 1 and 2, we provide the data from individual subjects. Table 1 summarises retinoscopy measurements at neutralisation, Retinomax measurements, bias measurements (retinoscopy-Retinomax measurements, using vector methods), and the vector dioptric difference $^{13}$ (VDD, the dioptric difference, averaged across all vector directions, taking into account sphere, cylinder and axis) for subjects in the retinoscopy group. Table 2 summarises subjective refinement measurements, Retinomax measurements, bias measurements (subjective refinement - Retinomax measurements, using vector methods), and the $\mathrm{VDD}^{13}$ for subjects in the subjective refinement group.

\section{REPRODUCIBILITY}

The dioptric deviations of each subject's Retinomax measured values of refractive error from the mean of each subject's Retinomax measured values are shown in Figure 1. As seen in Figure 1, one subject had an extreme outlier measured value of refractive error. This measured value was not included in the calculation of reproducibility for that subject. Based 
Table 1 Retinoscopy subjects

\begin{tabular}{|c|c|c|c|c|c|c|c|c|c|c|}
\hline \multirow[b]{2}{*}{ Subject } & \multicolumn{3}{|c|}{ Retinoscopy at neutralisation } & \multicolumn{3}{|c|}{ Retinomax representative } & \multicolumn{3}{|l|}{ Bias } & \multirow[b]{2}{*}{$V D D$} \\
\hline & $S p h$ & Cyl & Axis & $S p h$ & $C y l$ & Axis & $S p h$ & $C y l$ & Axis & \\
\hline 1 & 2.25 & 0.50 & 90 & 0.75 & 0.25 & 78 & 1.48 & 0.29 & 100 & 0.57 \\
\hline 2 & 3.00 & 0.00 & 26 & 1.50 & 0.50 & 99 & 1.00 & 0.50 & 9 & 0.41 \\
\hline 3 & 2.50 & 0.00 & & 0.25 & 0.25 & 112 & 2.00 & 0.25 & 22 & 1.27 \\
\hline 4 & 1.75 & 0.75 & 100 & 0.75 & 0.50 & 93 & 0.98 & 0.29 & 112 & 0.23 \\
\hline 5 & 3.50 & 0.00 & 96 & 2.75 & 0.50 & 103 & 0.25 & 0.50 & 13 & 1.12 \\
\hline 6 & 2.00 & 0.00 & 97 & 1.00 & 0.00 & 96 & 1.00 & 0.00 & & 0.35 \\
\hline 7 & 4.00 & 1.25 & 90 & 3.50 & 1.75 & 92 & -0.01 & 0.51 & 7 & 1.46 \\
\hline 8 & 3.00 & 0.50 & 90 & 0.25 & 0.25 & 112 & 2.69 & 0.36 & 76 & 2.32 \\
\hline 9 & 2.75 & 0.00 & 90 & 0.00 & 0.50 & 84 & 2.25 & 0.50 & 174 & 1.83 \\
\hline 10 & 3.00 & 0.00 & 64 & 1.75 & 0.50 & 14 & 0.75 & 0.50 & 104 & 0.45 \\
\hline 11 & 5.25 & 1.25 & 70 & 3.25 & 1.50 & 80 & 1.61 & 0.54 & 16 & 0.99 \\
\hline 12 & 2.75 & 0.25 & 95 & 1.50 & 0.25 & 90 & 1.23 & 0.04 & 137 & 0.09 \\
\hline 13 & 0.50 & 1.50 & 90 & -0.50 & 0.50 & 109 & 0.93 & 1.15 & 82 & 0.82 \\
\hline 14 & 2.50 & 0.50 & 90 & 2.00 & 0.25 & 81 & 0.49 & 0.27 & 98 & 0.88 \\
\hline 15 & -0.25 & 2.25 & 90 & -0.50 & 1.75 & 103 & -0.01 & 1.02 & 66 & 1.20 \\
\hline 16 & 0.50 & 2.25 & 90 & -1.00 & 2.50 & 84 & 1.10 & 0.56 & 145 & 0.44 \\
\hline 17 & 2.25 & 0.75 & 85 & 1.25 & 0.75 & 80 & 0.93 & 0.13 & 128 & 0.36 \\
\hline 18 & 3.00 & 1.00 & 95 & 1.75 & 0.75 & 97 & 1.25 & 0.26 & 89 & 0.22 \\
\hline 19 & 2.00 & 2.25 & 85 & 2.25 & 1.25 & 92 & -0.29 & 1.08 & 77 & 1.55 \\
\hline 20 & 0.75 & 0.00 & & -0.75 & 0.50 & 105 & 1.00 & 0.50 & 15 & 0.40 \\
\hline 21 & 2.50 & 1.00 & 85 & 1.25 & 0.75 & 92 & 1.21 & 0.33 & 68 & 0.24 \\
\hline 22 & 0.75 & 0.00 & 101 & -0.75 & 0.50 & 115 & 1.00 & 0.50 & 25 & 0.36 \\
\hline \multirow[t]{3}{*}{23} & 0.75 & 4.25 & 90 & 0.50 & 3.50 & 80 & -0.14 & 1.54 & 116 & 1.29 \\
\hline & & & & & & & & \multirow{2}{*}{\multicolumn{2}{|c|}{ Mean VDD }} & 0.82 \\
\hline & & & & & & & & & & 0.58 \\
\hline
\end{tabular}

$\mathrm{Sph}=$ sphere $; \mathrm{Cyl}=$ cylinder $;$ Bias $=$ retinoscopy measurement - Retinomax measurement, computed using vector methods ${ }^{13} ; \mathrm{VDD}$ $=$ vector dioptric difference ${ }^{13}$ (absolute value of the vector dioptric deviation between Retinomax representative and retinoscopy measurements, taking into account sphere, cylinder, and axis, calculated after mean bias was subtracted from retinoscopy measurements).

Table 2 Retinoscopy + subjective refinement subjects

\begin{tabular}{|c|c|c|c|c|c|c|c|c|c|c|}
\hline \multirow[b]{2}{*}{ Subject } & \multicolumn{3}{|c|}{$\begin{array}{l}\text { Retinoscopy and subject } \\
\text { refinement }\end{array}$} & \multicolumn{3}{|c|}{ Retinomax representative } & \multicolumn{3}{|l|}{ Bias } & \multirow[b]{2}{*}{$V D D$} \\
\hline & $S p h$ & $C y l$ & Axis & $S p h$ & $C y l$ & Axis & $S p h$ & $C y l$ & Axis & \\
\hline 1 & 0.25 & 0.00 & & -1.00 & 0.25 & 112 & 1.00 & 0.25 & 22 & 1.60 \\
\hline 2 & -1.50 & 1.75 & 75 & -1.25 & 1.75 & 76 & -0.28 & 0.06 & 31 & 0.36 \\
\hline 3 & 0.75 & 1.00 & 100 & 0.75 & 0.75 & 99 & -0.00 & 0.25 & 103 & 0.25 \\
\hline 4 & 0.25 & 1.25 & 70 & 0.25 & 0.50 & 72 & -0.00 & 0.75 & 69 & 0.73 \\
\hline 5 & 1.75 & 2.25 & 90 & 1.00 & 1.75 & 97 & -1.10 & 0.70 & 71 & 1.16 \\
\hline 6 & 1.75 & 2.50 & 100 & 2.25 & 2.00 & 95 & -0.57 & 0.63 & 117 & 0.54 \\
\hline 7 & 1.75 & 0.00 & 75 & 1.75 & 0.25 & 94 & -0.25 & 0.25 & 4 & 0.25 \\
\hline 8 & 0.75 & 1.75 & 70 & 1.75 & 1.25 & 74 & -1.02 & 0.54 & 61 & 1.11 \\
\hline 9 & 0.00 & 0.50 & 90 & 1.00 & 0.25 & 97 & -1.01 & 0.26 & 83 & 1.25 \\
\hline 10 & 0.75 & 0.00 & & 1.50 & 0.00 & & -0.75 & 0.00 & & 1.06 \\
\hline 11 & 0.75 & 1.25 & 85 & 1.75 & 1.00 & 92 & -1.06 & 0.37 & 65 & 1.26 \\
\hline 12 & -0.25 & 0.25 & 90 & 0.25 & 0.00 & & -0.50 & 0.25 & 90 & 0.56 \\
\hline 13 & 1.00 & 0.75 & 95 & 2.00 & 0.25 & 95 & -1.00 & 0.50 & 95 & 1.12 \\
\hline 14 & 2.25 & 0.75 & 90 & 1.25 & 0.75 & 98 & 0.90 & 0.21 & 49 & 1.42 \\
\hline 15 & 0.25 & 1.00 & 90 & 1.00 & 0.50 & 104 & -0.80 & 0.61 & 79 & 0.82 \\
\hline 16 & 1.00 & 0.50 & 90 & 1.00 & 0.50 & 101 & -0.10 & 0.19 & 51 & 0.11 \\
\hline 17 & 1.25 & 1.25 & 100 & 2.25 & 0.75 & 107 & -1.03 & 0.55 & 90 & 1.13 \\
\hline 18 & 2.25 & 0.75 & 85 & 0.50 & 0.50 & 105 & 1.63 & 0.49 & 64 & 2.67 \\
\hline 19 & -1.75 & 1.75 & 17 & -0.50 & 1.00 & 3 & -1.37 & 0.99 & 31 & 1.38 \\
\hline 20 & -1.25 & 4.25 & 100 & -0.75 & 3.75 & 96 & -0.62 & 0.75 & 122 & 0.58 \\
\hline 21 & 0.25 & 0.00 & & 1.25 & 0.00 & & -1.00 & 0.00 & & 1.41 \\
\hline \multirow[t]{3}{*}{22} & 0.50 & 1.75 & 105 & -1.00 & 2.00 & 112 & 1.11 & 0.52 & 49 & 1.97 \\
\hline & & & & & & & & \multicolumn{2}{|c|}{ Mean VDD } & 1.03 \\
\hline & & & & & & & & \multicolumn{2}{|l|}{ SD } & 0.58 \\
\hline
\end{tabular}

$\mathrm{Sph}=$ sphere $\mathrm{Cyl}=$ cylinder $;$ Bias $=$ subjective refinement measurement - Retinomax measurement, computed using vector methods $^{13} ;$ VDD $=$ vector dioptric difference ${ }^{13}$ (absolute value of the vector dioptric deviation between Retinomax representative and subjective refinement measurements, taking into account sphere, cylinder, and axis, calculated after mean bias was subtracted from subjective refinement measurements).

on comparisons of the subject's measured values and the subject's final representative measurement, it was clear that the Retinomax eliminated the outlier in selecting the representative measurement.

Measurements of reproducibility were obtained by calculating a mean deviation score for each subject. The grand mean of the deviation values across all subjects was $0.43 \mathrm{D}$ (SD 0.47). There was a significant correlation between reproducibility and age $\left(r_{\mathrm{s}}=-0.36\right.$, $\mathrm{p}<0.02)$; with older subjects, mean deviation scores were lower (that is, reproducibility of measurements was better). Reproducibility was not significantly related to the amount of spherical error or amount of cylinder.

We also evaluated Retinomax reproducibility for the retinoscopy and subjective refinement groups separately. For subjects who underwent retinoscopy, the mean deviation value was 0.49 $\mathrm{D}$ (SD 0.40). There was a significant correlation between reproducibility and age for the retinoscopy group $\left(r_{\mathrm{s}}=-0.49, \mathrm{p}<0.02\right)$; with older subjects, mean deviation scores were lower (that is, reproducibility of measurements was better). Reproducibility in the retinoscopy group was not significantly related to the amount of spherical error or amount of 


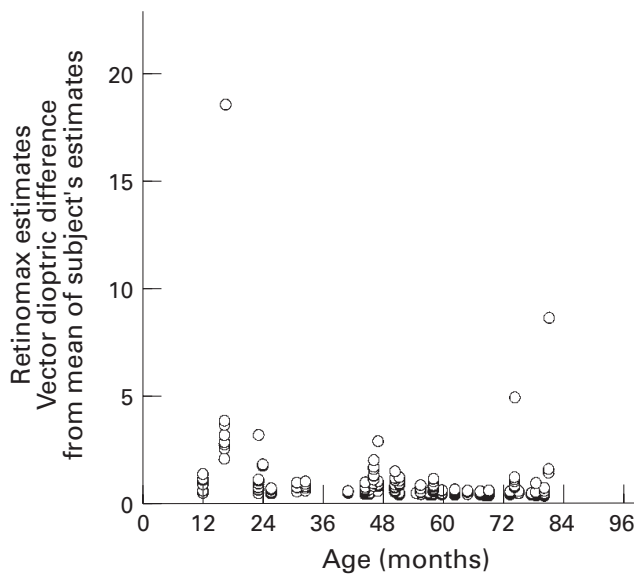

Figure 1 Individual subjects' Retinomax measured values plotted by age of subject. Circles represent the vector dioptric difference between each Retinomax estimate of a subject's refractive error and the average dioptric value ${ }^{13}$ of that subject's measured values. The Retinomax representative measurement of refractive error is not the mean of the individual measured values, as outliers are discarded.

cylinder. For subjects who were able to complete subjective refinement, the mean deviation value was $0.37 \mathrm{D}$ (SD 0.52). Reproducibility in the subjective refinement group was not significantly related to subject age, spherical power, or amount of cylinder. Reproducibility did not significantly differ between the retinoscopy and subjective refinement groups.

\section{MEASUREMENT BIAS}

Retinomax $v$ retinoscopy

The mean bias, calculated by subtracting Retinomax representative measurements from retinoscopy readings at neutralisation using the vector method of Harris, ${ }^{13}$ was $+1.19+0.11 \times$ 079. This estimate of bias includes any error introduced by the Retinomax, the retinoscopist, and average working distance (usually assumed to be two thirds of a metre, allowing for a $-1.5 \mathrm{D}$ retinoscopy lens). If we subtract the traditional $1.5 \mathrm{D}$ correction, bias for the Retinomax in comparison to standard retinoscopy was $-0.31+0.11 \times 079$.

Retinomax $v$ subjective refinement

The mean bias, calculated by subtracting Retinomax representative measurements from subjective refinement measurements using the vector method of Harris, ${ }^{13}$ was $-0.27+0.23 \times$ 072. This estimate of bias includes any error introduced by the Retinomax and by subjective refinement.

\section{MEASUREMENT AGREEMENT}

Retinomax $v$ retinoscopy

The mean of the absolute values of the dioptric deviations between subjects' Retinomax representative measurements and their retinoscopy measurements was $0.82 \mathrm{D}$ (SD 0.58). The dioptric deviation between Retinomax representative and retinoscopy measurements was not significantly related to subject age, amount of spherical power, or amount of cylinder.
Retinomax $v$ subjective refinement

The mean of the absolute values of the dioptric deviations between subjects' Retinomax representative measurements and their retinoscopy measurements was $1.03 \mathrm{D}$ (SD 0.59). The dioptric deviation between Retinomax representative and retinoscopy measurements was not significantly related to subject age, amount of spherical power, or amount of cylinder.

Agreement between Retinomax and retinoscopy and agreement between Retinomax and subjective refinement did not differ significantly.

\section{Discussion}

The Retinomax is a hand held instrument designed to provide a rapid estimate of refractive error. The portability and ease of use of the Retinomax suggest that it might be a useful tool for providing definitive 'gold standard' measurements of refractive error under cycloplegic conditions for use in research studies, and may be useful for screening young children for high refractive errors under noncycloplegic conditions.

We were able to achieve our goal of obtaining Retinomax measurements from all but two subjects between 1 and 8 years of age under cycloplegic conditions. Younger subjects tended to turn away from the Retinomax, as they do with any instrument that is placed close to the face. However, the ophthalmic technician was successful in obtaining Retinomax measurements in seven of nine children less than 3 years old. Retinoscopy was successfully completed in all children, and subjective refinement of the initial retinoscopy reading was successfully completed in 22 children.

The mean vector dioptric difference, taking into account sphere, cylinder, and axis, between the mean of each subject's Retinomax measured values of refractive error and the individual measured values obtained from that subject was $0.43 \mathrm{D}$ (SD 0.47). This reproducibility measure was correlated with subject age; there was better reproducibility of measurements with older subjects. Reproducibility did not differ between retinoscopy and subjective refinement groups. However, reproducibility was correlated with subject age in the retinoscopy group (younger and less cooperative children), but not in the subjective refinement group. These finding suggests that repeated Retinomax measurements or other methods of refraction should be used to determine the precise correction that should be prescribed in younger and less cooperative children, to allow for averaging of values.

It should be noted that the Retinomax provides a single representative measurements (or 'best estimate') based on the measured values of refractive error. Analysis of repeated representative measurements would most likely provide even better reproducibility than we found analysing repeated measured values.

Measurement bias for the Retinomax in comparison with retinoscopy phoropter readings at neutralisation (retinoscopy - Retinomax) was 
Table 3 Comparison of reproducibility of retinoscopy measurements ${ }^{10}$ with reproducibility of Retinomax measured values

\begin{tabular}{|c|c|c|c|c|}
\hline Study & Method & $\begin{array}{l}\text { Mean difference } \\
\text { (D) }\end{array}$ & $S D(N)$ & Range (D) \\
\hline \multicolumn{5}{|l|}{ Sphere } \\
\hline Safir et al $l^{10}$ & Retinoscopy (same retinoscopist) & 0.21 & $0.21(50)$ & $0-0.88$ \\
\hline Present study & Retinomax (1st two measured values) & 0.15 & $0.19(48)$ & $0-0.75$ \\
\hline \multicolumn{5}{|c|}{ 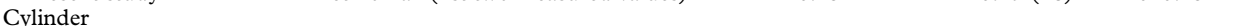 } \\
\hline Safir et al $l^{10}$ & Retinoscopy (same retinoscopist) & 0.18 & $0.16(50)$ & $0-0.53$ \\
\hline Present study & Retinomax (1st two measured values) & 0.18 & $0.17(48)$ & $0-0.75$ \\
\hline
\end{tabular}

*The absolute difference between two measurements was calculated (right eye data recalculated from tables in Safir $e t a l^{10}$ ).

$+1.19+0.11 \times 079$. If we subtract the traditional $1.5 \mathrm{D}$ correction from this value, bias for the Retinomax in comparison with standard retinoscopy was $-0.31+0.11 \times 079$. Measurement bias for the Retinomax in comparison with subjective refinement (subjective refinement Retinomax) was $-0.27+0.23 \times 072$. These data suggest that the Retinomax provided an average of approximately $0.25 \mathrm{D}$ less negative/more positive measures of refractive error than retinoscopy and subjective refinement.

The mean vector dioptric difference (averaged across all vector directions, taking into account sphere, cylinder, and axis) between unbiased retinoscopy measurements and their Retinomax representative measurements was $0.82 \mathrm{D}$ (SD 0.58). The mean vector dioptric difference (averaged across all vector directions) between subjective refinement measurements and their Retinomax representative measurements was $1.03 \mathrm{D}$ (SD 0.59). Thus, the accuracy (measurement agreement) of the Retinomax when compared with retinoscopy was similar to the accuracy of the Retinomax when compared with subjective refinement. In addition, the accuracy of the Retinomax, with reference to retinoscopy and subjective refinement, was not significantly related to subject age, spherical power, or amount of cylinder.

COMPARISON WITH PREVIOUS STUDIES:

MEASUREMENT REPRODUCIBILITY

Several previous studies have examined reproducibility of measurements of refractive error. In a study conducted by Safir et al, ${ }^{10}$ five ophthalmologists performed non-cycloplegic retinoscopy on 10 adult subjects on two separate occasions. The ophthalmologists were masked to the identity of the subjects at the time each retinoscopy was performed. Table 3 compares Safir et al's ${ }^{10}$ reproducibility of non-cycloplegic retinoscopy measurements of sphere and cylinder to reproducibility of the first two Retinomax measured values of sphere and cylinder for each subject in the present study. The results demonstrate little difference between reproducibility of measurements by the same retinoscopist in adult subjects (sphere $0.21 \mathrm{D}$; cylinder $0.18 \mathrm{D}$ ), and reproducibility of Retinomax measured values in children (sphere $0.15 \mathrm{D}$; cylinder $0.18 \mathrm{D}$ ).

Rosenfield and Chiu compared repeatability of non-cycloplegic subjective and objective refraction in 12 adult subjects. ${ }^{14}$ Each subject underwent five subjective refractive examinations over a 2 week period. Each examination was followed by objective refraction-that is, autorefraction, with the Canon Autoref R-1. The examiner was unaware of the results of the refractions. For Table 4, we reanalysed our data according to the methods of Rosenfield and Chiu, ${ }^{14}$ and compared the reproducibility results from Rosenfield and Chiu with the reproducibility of the first five Retinomax measured values in children. The results indicated poorer repeatability of Retinomax measured values in children, compared with subjective and objective refraction in adults. It should be noted, however, that each of the five objective measurements of refractive error for Rosenfield and Chiu's subjects was calculated as the mean of 25 autorefractor measurements. This method of determining objective refraction measurements may have obscured much of the variability in autorefractor measurements, providing an inappropriate comparison for repeatability of Retinomax measured values. In addition, one of our subjects had an extreme outlier Retinomax measured value, which contributed to the large mean SD for sphere and spherical equivalent (see Fig 1). When the Retinomax data are analysed with-

Table 4 Comparison of reproducibility of subjective and objective* refraction in adults ${ }^{14}$ with reproducibility of Retinomax measured valuest in children

\begin{tabular}{|c|c|c|c|c|c|}
\hline & Method & Study & Subjects & Mean $S D(D)$ & $95 \%$ limits (D) \\
\hline \multirow[t]{3}{*}{ Sphere } & Subjective refraction & Rosenfield and & Adult $(\mathrm{n}=12)$ & 0.14 & $+/-0.27$ \\
\hline & Objective refraction & Chiu $^{14}$ & & 0.16 & $+/-0.31$ \\
\hline & Retinomax measured values & Present study & Child $(n=43)$ & 0.42 & $+/-0.82$ \\
\hline \multirow[t]{3}{*}{ Cylinder } & Subjective refraction & Rosenfield and & Adult $(\mathrm{n}=12)$ & 0.08 & $+/-0.16$ \\
\hline & Objective refraction & Chiu $^{14}$ & & 0.19 & $+/-0.37$ \\
\hline & Retinomax measured values & Present study & Child $(n=43)$ & 0.24 & $+/-0.47$ \\
\hline \multirow[t]{3}{*}{ Spherical equivalent } & Subjective refraction & Rosenfield and & Adult $(\mathrm{n}=12)$ & 0.15 & $+/-0.29$ \\
\hline & Objective refraction & $\mathrm{Chiu}^{14}$ & & 0.14 & $+/-0.27$ \\
\hline & Retinomax measured values & Present study & Child $(n=43)$ & 0.39 & $+/-0.76$ \\
\hline
\end{tabular}

Mean SD = standard deviation of each subject's five measurements of the right eye was determined, and the mean of the standard deviations was calculated.

$95 \%$ Limits $=1.96$ times the mean SD.

^Following each of five subjective refractions, each subject had 25 measurements taken with the Canon R-1 autorefractor. Each of the five measured values of objective refraction were calculated as the mean of 25 measurements.

†The first five Retinomax measured values on each subject were analysed according to the methods of Rosenfield and Chiu. ${ }^{14}$ Only subjects with at least five measured values were included (43/45). 
Table 5 Comparison of reproducibility of retinoscopy, subjective refraction, and autorefraction measurements in adults ${ }^{11}$ with reproducibility of Retinomax measured values in children

\begin{tabular}{lllcll}
\hline Method & Study & Sample (n) & $\begin{array}{l}\star_{\text {Mean difference }} \\
(D)\end{array}$ & $\begin{array}{l}\text { Standard } \\
\text { deviation (D) }\end{array}$ & $\begin{array}{l}\text { 95\% Confidence } \\
\text { interval (D) }\end{array}$ \\
\hline Retinoscopy & Zadnik et $a l^{11}$ & Adults (40) & 0.07 & 0.48 & -0.87 to 1.02 \\
Subjective refraction & Zadnik et al ${ }^{11}$ & Adults (40) & -0.01 & 0.48 & -0.95 to 0.93 \\
Autorefraction & Zadnik et al ${ }^{11}$ & Adults (40) & 0.05 & 0.16 & -0.27 to 0.37 \\
Retinomax & Present study & Children (35) & -0.06 & 0.21 & -0.47 to 0.35 \\
\hline
\end{tabular}

${ }^{\star}$ Mean of the differences between two measurements in the vertical meridian (right eye only).

†For each subject, the difference (D) between the first two measured values of spherical power in the vertical meridian was determined. Subjects with oblique axes of astigmatism (that is, greater than $20^{\circ}$ from horizontal or vertical) were not included in these analyses.

Table 6 Agreement between subjective refraction and autorefraction measurements in adults ${ }^{15}$ and agreement between retinoscopy and Retinomax representative measurements in children

\begin{tabular}{llllll}
\hline Method & Study & Sample (n) & $\begin{array}{l}\text { Mean } \\
\text { difference } \\
(D) \star\end{array}$ & $\begin{array}{l}\text { Standard } \\
\text { deviation } \\
(D)\end{array}$ & $\begin{array}{l}\text { 95\% Limits of } \\
\text { agreement (D)t }\end{array}$ \\
\hline (Humphrey 500) - (subjective refraction) & Kinge et al ${ }^{15}$ & Adults (224) & -0.23 & 0.47 & -1.18 to +0.71 \\
(Nidek AR-1000)-(subjective refraction) & Kinge t al ${ }^{15}$ & Adults (80) & -0.13 & 0.27 & -0.68 to 0.41 \\
(Retinomax) - (retinoscopy) $\ddagger$ & Present study & Children (23) & +0.26 & 0.66 & -1.06 to 1.58 \\
(Retinomax) - (subjective refinement) & Present study & Children (22) & +0.07 & 0.84 & -1.61 to 1.75 \\
\hline
\end{tabular}

${ }^{\star}$ Mean of the differences between two measurements of spherical equivalent (right eye only).

†Mean \pm 2 standard deviations.

$\ddagger$ Standard 1.5 D correction applied to retinoscopy measurements (phoropter at neutralisation) before calculations.

out that subject, the mean SDs are 0.26 for sphere, 0.22 for cylinder, and 0.22 for spherical equivalent. Nevertheless, Rosenfield and Chiu's results also indicated that subjective refraction in adults yielded better reproducibility than Retinomax measured values in children.

Zadnik and colleagues conducted a study examining the reproducibility of cycloplegic retinoscopy, subjective refraction, and autorefraction in 40 adult subjects. ${ }^{11}$ For Table 5, we reanalysed our data according to the methods of Zadnik et al, and compared the results from Zadnik et al with the reproducibility of the first two Retinomax measured values in children. The findings were similar to the results of our comparisons with Safir et al's ${ }^{10}$ data. There was little difference in reproducibility of Retinomax measured values in children $(-0.06 \mathrm{D})$ and reproducibility of retinoscopy $(0.07 \mathrm{D})$, subjective refraction $(-0.01 \mathrm{D})$, and autorefraction $(0.05 \mathrm{D})$ in adults.

\section{COMPARISON WITH PREVIOUS STUDIES:}

MEASUREMENT AGREEMENT

In a recent study, Kinge and colleagues ${ }^{15}$ evaluated agreement between subjective refraction and autorefraction in adult subjects made cycloplegic. For Table 6, we analysed our data according to the method used by Kinge et al. ${ }^{15}$ The Retinomax and retinoscopy agreement was comparable with agreement between the Humphrey autorefractor and subjective refraction, and poorer than agreement between the Nidek autorefractor and subjective refraction. The Retinomax and subjective refinement had better agreement (mean difference closer to 0) than both autorefractors (Humphrey and Nidek) and subjective refraction. These analyses suggest that the Retinomax provides agreement with retinoscopy and subjective refinement in young children that is comparable with agreement between other autorefractors (Humphrey and Nidek) and subjective refraction in adults.
Although the magnitude of the mean differences between the three autorefractors and subjective refraction/retinoscopy were similar (ranging from $0.07 \mathrm{D}$ to $0.26 \mathrm{D}$ ), the sign of the mean differences differed. That is, the Retinomax provided more positive/less negative measurements, in comparison with retinoscopy and subjective refinement, and the Humphrey and Nidek autorefractors provided more negative/less positive measurements, in comparison with subjective refraction.

\section{Conclusions}

The data from the present study indicate that the reproducibility of the Retinomax is similar to that reported for cycloplegic and noncycloplegic retinoscopy, subjective refraction, and autorefraction. Although our results indicate that the Retinomax provides consistent and accurate measured values of refractive errors under cycloplegic conditions, the variability in measurements is large enough to suggest that repeated Retinomax measurements or other methods should be used to determine the precise correction that should be prescribed for very young children.

The Retinomax may be a useful tool for obtaining definitive measurements of refractive error under cycloplegic conditions. However, the present study suggests that the Retinomax provides approximately $0.25 \mathrm{D}$ more positive/ less negative measurements of refractive error than subjective refinement in children. We did not have the power to determine if this mean differs significantly from zero.

The results of the present study suggest that the Retinomax might be used successfully as a screening tool, as we were able to obtain measurements from the majority $(96 \%)$ of the children in our study. The Retinomax requires very little training and practice to be used effectively, and it provides immediate printouts of measurements. These features would allow examiners in screening situations to identify children with significant refractive errors, 
explain the findings to the parents, and refer or schedule the child for a follow up examination. However, before widespread use of the Retinomax for refractive error screening, additional research would be required to evaluate the reproducibility and accuracy of the Retinomax under non-cycloplegic conditions, as cycloplegia is often impractical in screening settings.

This research was presented in part at the Association for Research in Vision and Ophthalmology Annual Meeting, Ft Lauderdale, Florida, 24 April 1996.

This study was supported by NIH grant EY05804 to Velma Dobson, $\mathrm{PhD}$, and by a grant from The Whitaker Foundation to Joseph M Miller, MD. The authors have no proprietary interest in the present work.

The authors thank David Henderson and Nikon, Inc, for the use of the Retinomax.

1 Freeman RD, Mitchell DE, Millodot M. A neural effect of partial visual deprivation in human. Science 1972;175:1384-6.

2 Mitchell DE, Freeman RD, Millodot M, Haegerstrom G. Meridional amblyopia: evidence for modification of the human visual system by early visual experience. Vision Res 1973;13:535-58.

3 Ingram RM, Traynar MJ, Walker C, Wilson JM. Screening Ingram RM, Traynar MJ, Walker C, Wilson JM. Screening
for refractive errors at age 1 year: a pilot study. $B r \quad \mathscr{J}$ for refractive errors at age
Ophthalmol 1979;63:243-50.

4 Gwiazda J, Bauer J, Thorn F, Held R. Meridional amblyopia does result from astigmatism in early childhood. Clin Vis Sci 1986;1:145-52.
5 Cobb SR, MacDonald CF. Resolution acuity in astigmats: evidence for a critical period in the human visual system. $\mathrm{Br}$ f Physiol Opt 1978;32:38-49.

6 Mohindra I, Jacobson SG, Held R. Binocular visual form deprivation in human infants. Doc Ophthalmol 1983;55: 237-49.

7 Atkinson J, Braddick O, Wattam-Bell J, Watson P, Durden $\mathrm{K}$, Bobier W, et al. The prediction and prevention of strabismus and amblyopia achieved in the Cambridge infant photorefraction screening programme. Ophthal Physiol Opt 1989;9:467.

8 Lithander J, Sjöstrand J. Anisometropic and strabismic amblyopia in the age group 2 years and above: a prospective study of the results of treatment. Brf Ophthalmol 1991;75. 111-6.

9 Hopkisson B, Arnold P, Billingham B, McGarrigle M, Shribman S. Can retinoscopy be used to screen infants for amblyopia? A longitudinal study of refraction in the first year of life. Eye 1992;6:607-9.

10 Safir A, Hyams L, Philpot J, Jagerman LS. Studies in refraction. I The precision of retinoscopy. Arch Ophthalmol 1970; 84:49-61.

11 Zadnik K, Mutti DO, Adams AJ. The repeatability of measurements of the ocular components. Invest Ophthalmol Vis Sci 1992;33:2325-33.

12 Long WF. A matrix formalism for decentration problems. Am f Optom Physiol Opt 1976;53:27-33.

13 Harris WF. Algebra of sphero-cylinders and refractive errors, and their means, variance and standard deviation. Am 7 Optom Phys Optics 1988;65:794-802.

14 Rosenfield $M$, Chiu NN. Repeatability of subjective and objective refraction. Optom Vis Sci 1995;72:577-9.

15 Kinge B, Midelfart A, Jacobsen G. Clinical evaluation of the Allergan Humphrey 500 autorefractor and the Nidek AR-1000 autorefractor. Br f Ophthalmol 1996;80:35-9. 